\title{
SPREAD PATTERn ANALYSis TOOL (SPAT): II. EXAMPLES OF AirCRAFT PATTERN ANALYSIS
}

\author{
T. E. Grift, J. T. Walker, D. R. Gardisser
}

\begin{abstract}
The quality of aerial spread patterns was studied based on over 400 test runs in Arkansas during the period 1992-1997. A custom developed computer program called SPAT (Spread Pattern Analysis Tool, Grift, 2000) was used to compute overlapped spread patterns and to determine the pattern quality. The quality of a spread pattern is traditionally expressed by the average application rate (the mean of the overlapped pattern) and uniformity [expressed in the statistical coefficient of variation (cv)]. Both parameters depend highly on the swath width. Inspired by studying the cv-swath width relationships, a new measure for pattern quality called "robustness" was conceived. This parameter indicates the flexibility of a spread pattern shape, or the ability of the applicator to vary the swath width (and, hence, the application rate), either purposely or due to unintended flight path errors, and be confident that the overlapped pattern will have an acceptable uniformity. Grift (2000) stated that, as a rule of thumb, patterns with a robustness factor lower than 5\% can be considered robust. From studying spread patterns of unadjusted spreaders in Back\&Forth mode, it was concluded that only $3.45 \%$ of them are robust and after adjustments had been made, $2.75 \%$. In RaceTrack mode, the percentage of robust patterns was $8.59 \%$ and after adjustments it increased to $10.28 \%$. The majority of all patterns (approximately $70 \%$ ), in both modes, had robustness factors between 5\% and 15\%. The results of this study imply a definite need to improve the quality of aerial application spread patterns by (1) avoiding pattern shapes that are sensitive to transforming to nonrobust, and (2) periodic calibration. The magnitude of the problem indicates that periodic adjustment of spreaders will not be sufficient, redesign of equipment and spreading procedures may be necessary to accomplish overall high quality spread patterns.
\end{abstract}

Keywords. Aerial spread patterns, Granular fertilizer application, Spread pattern robustness.

$\mathrm{T}$ he quality of spread patterns in the field is determined to a large extent by the performance of the aircraft-spreader combination. Deviations from the ideal uniform application pattern can lead to "streaking", resulting in yield loss and undesired environmental side effects (Helms et al., 1987). To ensure the quality of fertilizer application, the Cooperative Extension Service of the University of Arkansas organizes calibration workshops, where aerial applicators have their equipment tested and, if necessary, adjusted. Two modes of operation are in use in aerial fertilizer application, Back\&Forth (the most popular) and Racetrack, as schematically shown in figure 1.

The causes for non-uniform spread patterns are varied. The fertilizer material is contained in a hopper, mounted within the fuselage of the aircraft. Under the influence of severe vibrations which are present in aircraft, the material

Article was submitted for publication in March 2000; reviewed and approved for publication by the Power \& Machinery Division of ASAE in August 2000.

The authors Tony E. Grift, ASAE Member Engineer, Assistant Professor, Department of Biosystems Engineering, Auburn University, Auburn, Alabama; Joel T. Walker, ASAE Member Engineer, Professor, Department of Biological and Agricultural Engineering, University of Arkansas, Fayetteville, Arkansas; and Dennis R. Gardisser, ASAE Member Engineer, Extension Agricultural Engineer, Cooperative Extension Service, University of Arkansas, Little Rock, Arkansas. Corresponding author: Tony Grift, Auburn University, Auburn, Biosystems Engineering Dept., 200 Corley Bldg., Auburn, AL 368495417, phone: 334.844.3545, fax: 334.844.3530, e-mail: <tegrift@eng.auburn.edu>.

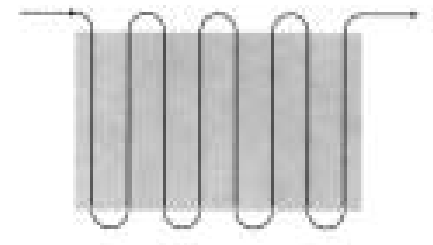

Backefont

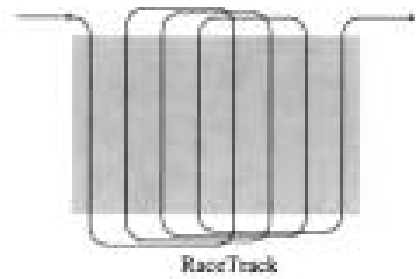

Figure 1-Back\&Forth and RaceTrack flight paths.

segregates which implies a particle size distribution change during the spreading operation.

Parish (1999) has shown that the fill level of the hopper has a distinct effect on the delivery rate of ground-based broadcast spreaders, and the same can be expected in aerial application. Both these phenomena have had surprisingly little attention in research.

When the material leaves the hopper, it is forced through the gate into the throat of the spreader. The gate can be a major source of "skewness" in the pattern if it does not open evenly.

When there is not enough energy available to the fertilizer mass flow, spreader overload occurs. This usually results in a pattern with a narrow spread width and a high application rate in the center.

Eventually, particles fall to the ground at a constant velocity in a situation where the drag force and gravity force are in equilibrium. During this process, side wind can 
have a negative effect on the spread pattern, especially in Back\&Forth operation.

During testing, often an aircraft produces a spread pattern with an acceptable cv at a certain swath width, but the rate is off. Also the opposite happens, at a certain swath width the rate is satisfactory but the $\mathrm{cv}$ is unacceptable. One of the causes of this problem is the fact that applicators have traditionally had little control over the fertilizer output of the spreader. To alleviate this problem, Grift and Hofstee (1997) have developed a sensor that can be used to measure the fertilizer mass flow in the spreader ducts. An efficient spreading approach would be to have the spreader always work at the maximum material flow (without overloading it) and adjust the swath width to set the application rate. This approach is only valid if there is a guarantee that the uniformity is acceptable, even when the swath width is changed. Also, when pilots make errors in flight paths, this pattern would be "forgiving". A pattern that has acceptable uniformity for a large range of swath widths is called "robust".

In summary, there are many factors that can negatively influence the spread pattern quality. Before any improvements can be made in controllable influences, it is necessary to define a high quality spread pattern and to identify what causes low quality spread patterns.

The objectives of this study were to:

- Analyze the robustness of real life spread patterns.

- Identify potential causes of non-robustness.

- Investigate the effect of calibration on robustness.

\section{MATERIALS AND METHODS}

The spread patterns were obtained using urea fertilizer according to ASAE Standard S386.2 (1999). The test arrangement consisted of 25 funnel-shaped collection trays, each with an area of $1 \mathrm{~m}^{2}$, aligned equidistantly at a distance of $1.8 \mathrm{~m}$. The collection line was placed perpendicular to the wind direction and the flight path was marked with flags, perpendicular to the collection line, to avoid side wind effects. The ground speed of the aircraft and the height of the spreader were measured along with ambient conditions, temperature, humidity, wind velocity, and direction. After the run, the trays were emptied and the contents weighed. The actual data (weights) from each run were stored in Excel worksheets along with test information such as the date, run number, pilot's name, aircraft-spreader type, and the target rate.

\section{DEFINITION OF RoBuSTNESS}

The overall quality of a spread pattern can be expressed in many ways. The applicator's objective is to disperse fertilizer in such a way that (1) the overlapped pattern is of acceptable uniformity, (2) the applied rate is close to the target rate, and (3) a large spread width is obtained. These quantities are usually contradictory and, therefore, priorities must be set.

The uniformity of an overlapped pattern must be acceptable, to avoid damage to the crop. A rule of thumb, commonly used in aerial application is that a coefficient of variation lower than $15 \%$ will prevent damage (Gardisser, 1993). Although other values may be appropriate for different crops, materials and environmental conditions, for reasons of comparison, the value of $15 \%$ was used throughout the analyses in this article.

The applied rate is also important, although deviations from the rate are not as prominently visible as uniformity variations, which often cause discoloration of the crop (streaks) that indicate over application or under application. A large swath width is of less importance, although it does have an effect on the efficiency of operation by minimizing the total travel distance.

Uniformity can be studied from cv-swath width curves. An example is shown in figure 2 , for a high quality spread pattern. The horizontal line in the graph represents the $15 \%$ rule of thumb.

One way to quantify the quality of a spread pattern would be to report the maximum swath width where the cv is lower than $15 \%$ (in this case around $37 \mathrm{~m}$ ). This would, however, wrongly imply that all smaller aircraft produce lower quality patterns than large ones, because their less powerful engines do not allow for larger swaths. Also, some patterns have a small range of higher swath widths where the cv is lower than $15 \%$ and a distinct area in the lower swath width range where the cv is higher than $15 \%$. These patterns would be of high quality according to this definition, which is not justifiable. The previous arguments show that the quality of a spread pattern should take a range rather than a single swath width into account. An intuitive choice would be to take the average $\mathrm{cv}$ for swath widths from zero to the intersect with the $15 \%$ line. This method has disadvantages for patterns which cv-swath width curve is as shown in figure 3 .

The cv swath width curve as shown in figure 3 is typical for skewed spread patterns. When the average cv would be

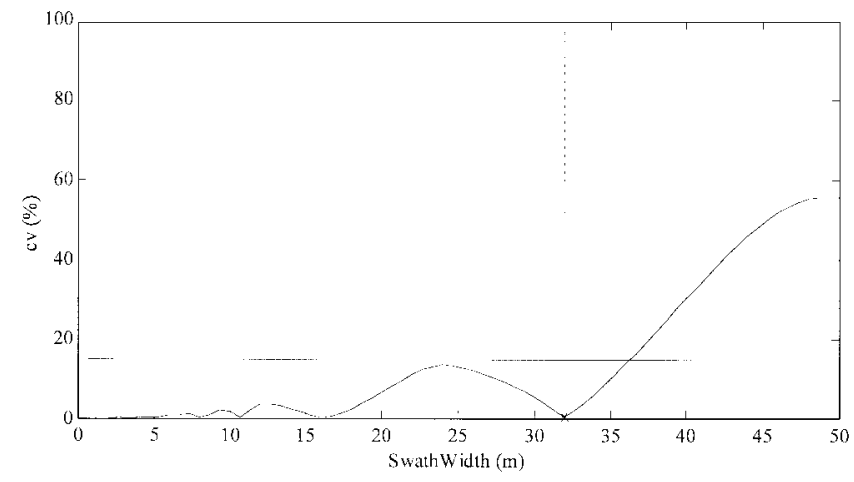

Figure 2-The cv-swath width curve of a high quality spread pattern.

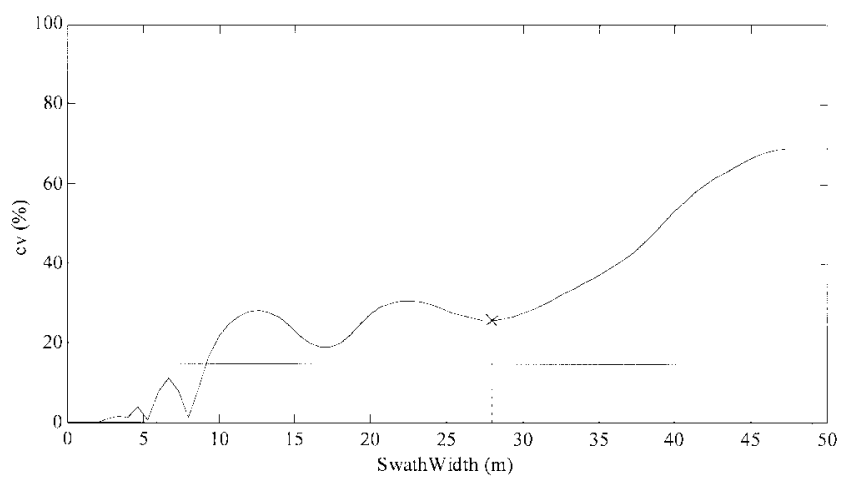

Figure 3-The cv-swath width curve of a low quality spread pattern. 
taken here from zero to the intersect with the $15 \%$ line (here at approximately $9 \mathrm{~m}$ swath width), it would give a very low value for the quality factor, and judge this an excellent pattern. This is obviously not the case, since the majority of swath widths have very high cvs. Therefore, another definition was used, which takes the average cv from zero to the minimum at the maximum swath width (here at approximately $27 \mathrm{~m}$ ). This effectively excludes the range to the right of the minimum at $27 \mathrm{~m}$, which is valid, since this is a range where the $\mathrm{cv}$ monotonically increases to a constant value. This is a feature that is shared by any spread pattern which makes the criterion universal. Based on the $15 \%$ rule of thumb, a spread pattern can be considered robust when the robustness factors in Back\&Forth mode (AcvBF) and RaceTrack mode (AcvRT) are lower than 5\% (Grift, 2000).

\section{Spread Pattern Analysis Tool (SPAT)}

For analysis and visualization of the test results, a custom developed Windows-based Spread Pattern Analysis Tool (SPAT) was used (Grift, 2000). This program produces overlapped patterns in Back\&Forth and RaceTrack modes, as well as cv-swath width and rateswath width curves. It also computes the robustness factors AcvBF and AcvRT (Average cv in Back\&Forth and RaceTrack mode respectively). The program allows for centering spread patterns, to correct for off-center aircraft passages during tests. It has built-in interpolation of data to smooth the output plots and can also be used to compare consecutive runs to study improvements after adjustments. After the program has computed an overlapped pattern (in Back\&Forth or RaceTrack mode) the user can select a swath width and study the overlapped pattern and pattern characteristics.

SPAT was developed for aerial application, but it can be used for ground-based application as well.

\section{Analysis of Realistic SpREad Patterns}

Four test runs were selected to illustrate the robustness concept and to investigate the potential causes for nonuniformity. Figures 4 through 7, represent the SPAT output for four realistic spread patterns that are illustrative in showing the causes of non-robustness. The figures 4 through 7 are divided into four subplots each. The subplots $A$ and $B$ show the overlapped pattern in Back\&Forth mode, and the subplots $\mathrm{C}$ and D in RaceTrack mode. The subplots $\mathrm{A}$ and $\mathrm{C}$ show the pattern with a rate close to the target rate, and the subplots $\mathrm{B}$ and $\mathrm{D}$ show the pattern in optimal uniformity/swath width combination (minimum $\mathrm{cv}$ at the maximum swath width). The first example (fig. 4), shows a spread pattern that has a smooth triangular shape.

The cv-swath width curve shows a low cv for swath widths ranging from zero to approximately $25 \mathrm{~m}$ for both Back\&Forth and RaceTrack modes. In general, the cvswath width curves of symmetrical spread patterns are the same, which is why the cv-swath width curves for Back\&Forth and RaceTrack mode are very similar for this near-symmetrical shape. According to the cv swath width curve, the applicator can adjust the application rate by flying at any swath width lower than $25 \mathrm{~m}$ without having to worry about non-uniformity. A pattern that exhibits this flexibility is called robust, indicated by the low values of AcvBF (2.82\%) and AcvRT (2.42\%).

The second example, best characterized as a wide trapezoidal spread pattern, is shown in figure 5 .

A trapezoidal shape is often desired because, when properly overlapped, it can achieve almost perfect uniformity. The correct rate can be obtained by having the target rate at the horizontal leg. This pattern seems quite good, shown at a swath width of $27 \mathrm{~m}$ (subplots B and D), but the target rate of $112\left(\mathrm{~kg} \mathrm{ha}^{-1}\right)$ is not obtained here [it is $\left.89\left(\mathrm{~kg} \mathrm{ha}^{-1}\right)\right]$. From the rate-swath width curve, it is clear that the swath width must be reduced to $21.6 \mathrm{~m}$ to obtain a rate close to the target rate. However, when the swath width is changed to $21.6 \mathrm{~m}$ (see subplots $\mathrm{A}$ and $\mathrm{C}$ ), the rate is acceptable $\left(110 \mathrm{~kg} \mathrm{ha}^{-1}\right)$, but the $\mathrm{cv}$ is $18 \%$ (Back\&Forth) and 19\% (RaceTrack). This trade-off between rate and uniformity is often seen in practice.

The example illustrates that the "shoulders" of the trapezoidal pattern cause a region of non-uniformity at a swath width range of approximately 17 to $24 \mathrm{~m}$. In general, when the horizontal leg of the trapezoid is smaller than one-third of the total spread width, the "hump" in the cvswath width curve stays under $15 \%$. This pattern shape is called the critical trapezoid (Grift, 2000). The robustness factors, AcvBF and AcvRT, are 7.82\% and 7.99\%, respectively, and both are higher than $5 \%$. The pattern is non-robust in Back\&Forth as well as RaceTrack mode.

The third example, shown in figure 6, shows a pattern similar to the previous wide trapezoidal pattern, although in this case, the left shoulder of the pattern is higher than the right one. This phenomenon, called skewness, is only mildly apparent in this pattern, but, as will be shown, the effects are quite pronounced.

A skewed pattern results in different $\mathrm{cv}$-swath width curves for RaceTrack and Back\&Forth modes (compare subplots A and B to subplots C and D). In general, skewness increases the cv for all swath widths, particularly in Back\&Forth mode. The subplots B and D show that at a swath width of $25.2 \mathrm{~m}$, the pattern is on the uniformity limit (15\%) for Back\&Forth and slightly under the limit for RaceTrack mode (12.8\%). In both modes, deviations from the $25.2 \mathrm{~m}$ flight path will result in unacceptable uniformity. The causes of skewness are:

1. Poorly adjusted spreaders. Vanes in the inlet (and sometimes outlet) can be adjusted which can lead to asymmetry in the particle flow cloud behind the aircraft.

2. Faulty spreader gates that do not open evenly across the whole width.

3. Side wind, since smaller particles are more affected than larger ones.

The robustness factors, AcvBF and AcvRT, are 12.21\% and $10.37 \%$, respectively, and both are higher than $5 \%$. This renders the pattern non-robust in Back\&Forth and RaceTrack mode.

The last pattern, shown in figure 7, is a classic M-shape. This pattern is sometimes seen in ground based pendulum spreaders (Parish, 1999). In aerial application, M-shape patterns are often seen when too much emphasis is placed on a wide swath and too much material is fed into the outer vanes. This trend has been fueled by the manufacturers' tendency to add more vanes to larger spreaders, mounted on aircraft with more powerful (turbofan) engines. Bansal 
Elles Simulation Prelerences Mode Help
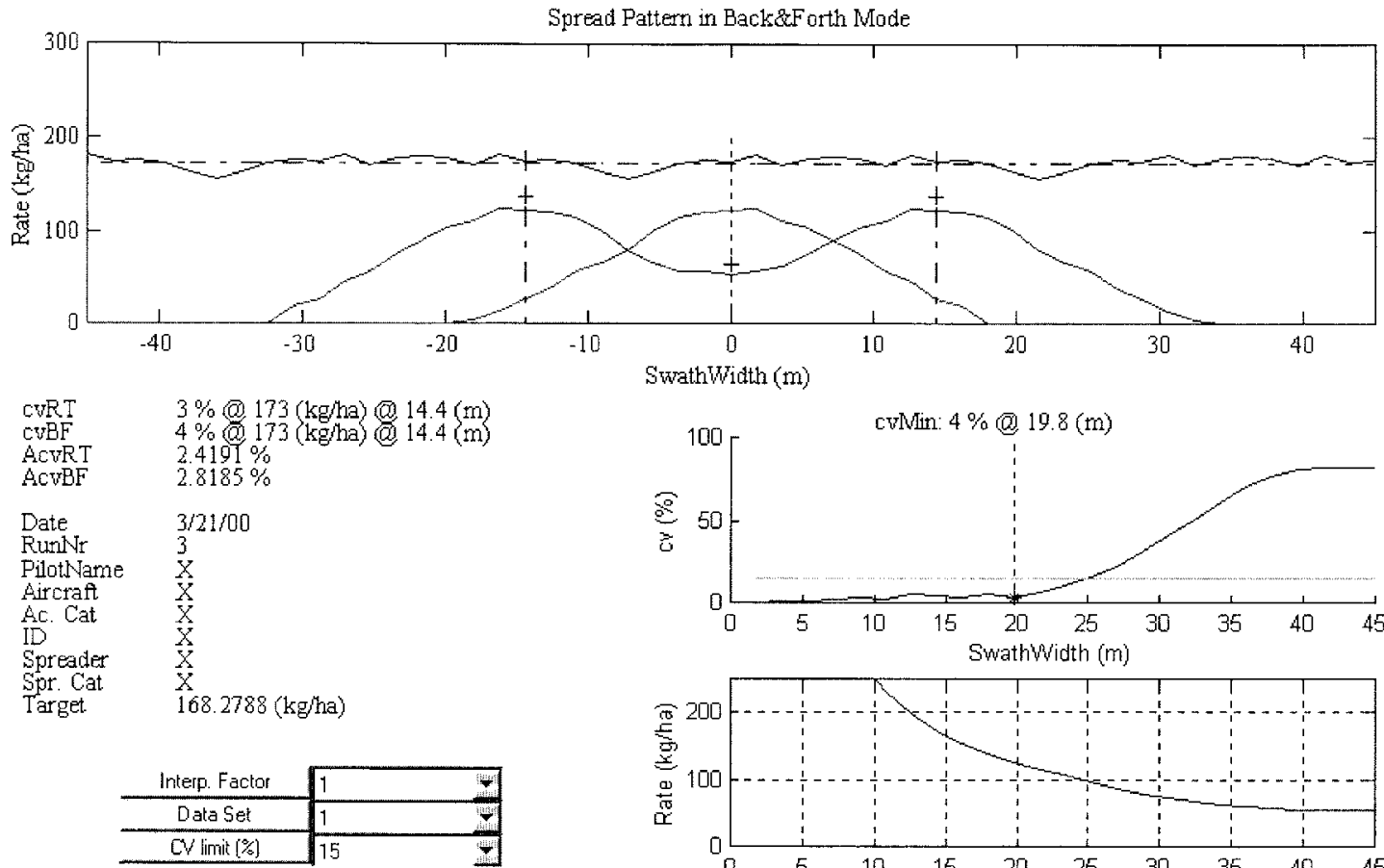

SwathWidth (m)
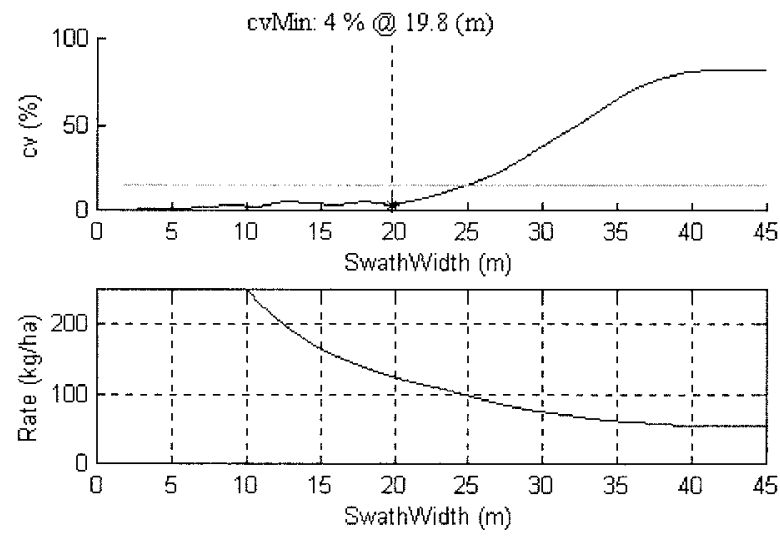

Figure 4(a)-Characteristics of a smooth triangular spread pattern (Back\&Forth, rate close to target rate).

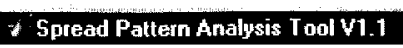

Elles Simulation Preferences Mode Help

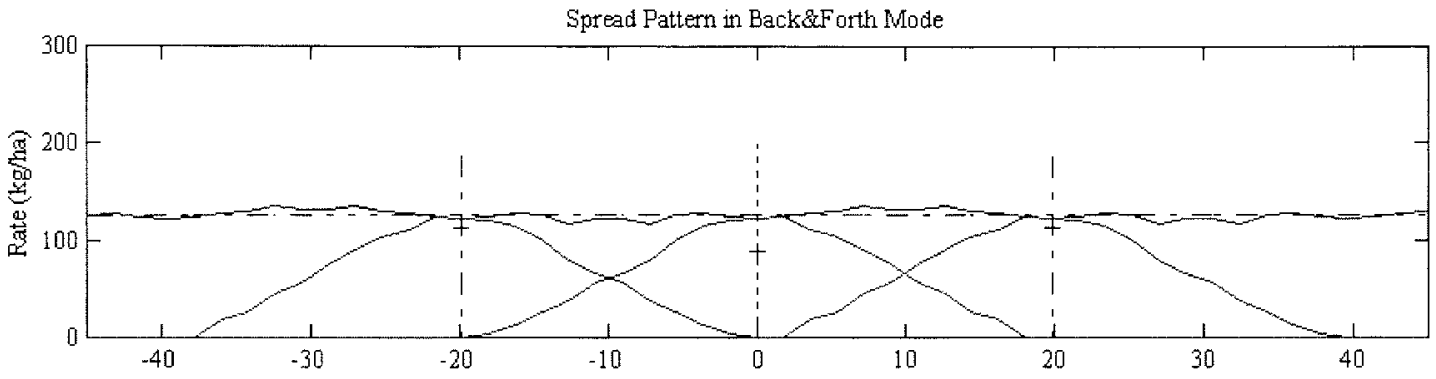

$\begin{array}{ll}\text { cURT } & 3 \% @ 126(\mathrm{~kg} / \mathrm{ha}) 19.8(\mathrm{~m}) \\ \text { cWBF } & 4 \%(126(\mathrm{~kg} / \mathrm{ha}) 19.8(\mathrm{~m})\end{array}$

$\begin{array}{ll}\text { ACvRT } & 2.4191 \% \\ \text { ACvBF } & 2.8185 \%\end{array}$

Date $\quad 3 / 21 / 00$

Runkir

PilotName

Aircraft

Ac. Cat

ID

Spreader

Spr. Cat $\quad X$

Target $168.2788(\mathrm{~kg} / \mathrm{ha})$

\begin{tabular}{|c|c|c|}
\hline Interp. Factor & 1 & -7 \\
\hline Data Set & 1 & (1): \\
\hline CV limit [s] & 15 & 7 \\
\hline
\end{tabular}

SwathWidth (m)
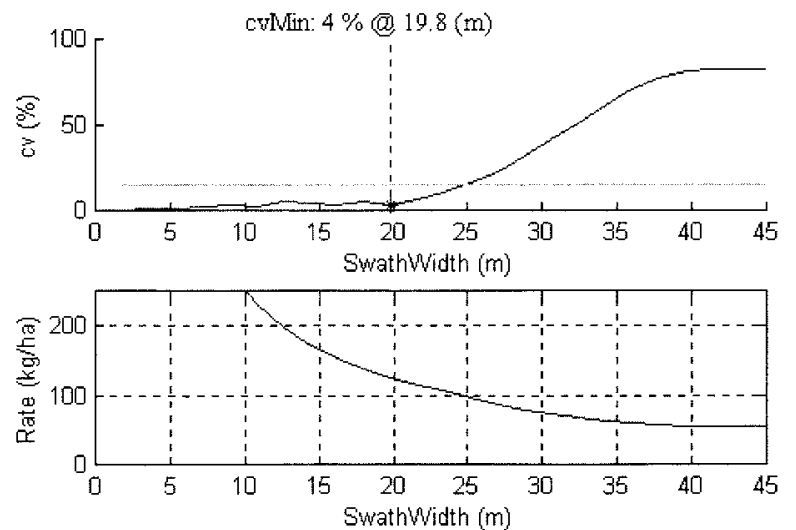

Figure 4(b)-Characteristics of a smooth triangular spread pattern (Back\&Forth, optimal uniformity/swath width combination). 

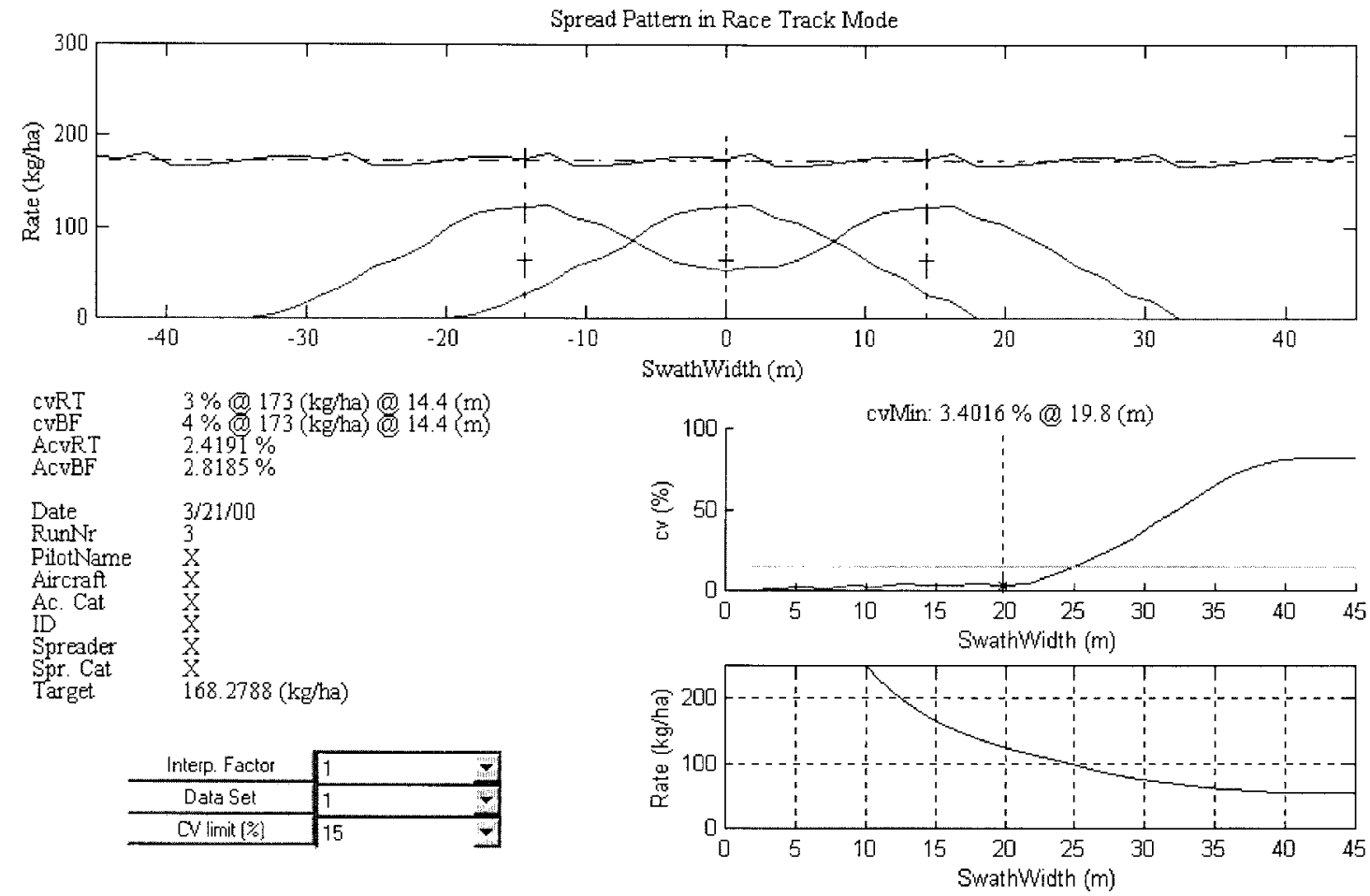

Figure 4(c)-Characteristics of a smooth triangular spread pattern (RaceTrack, rate close to target rate).

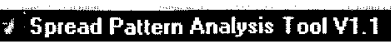

Files Simulation Ereferences Wode Help
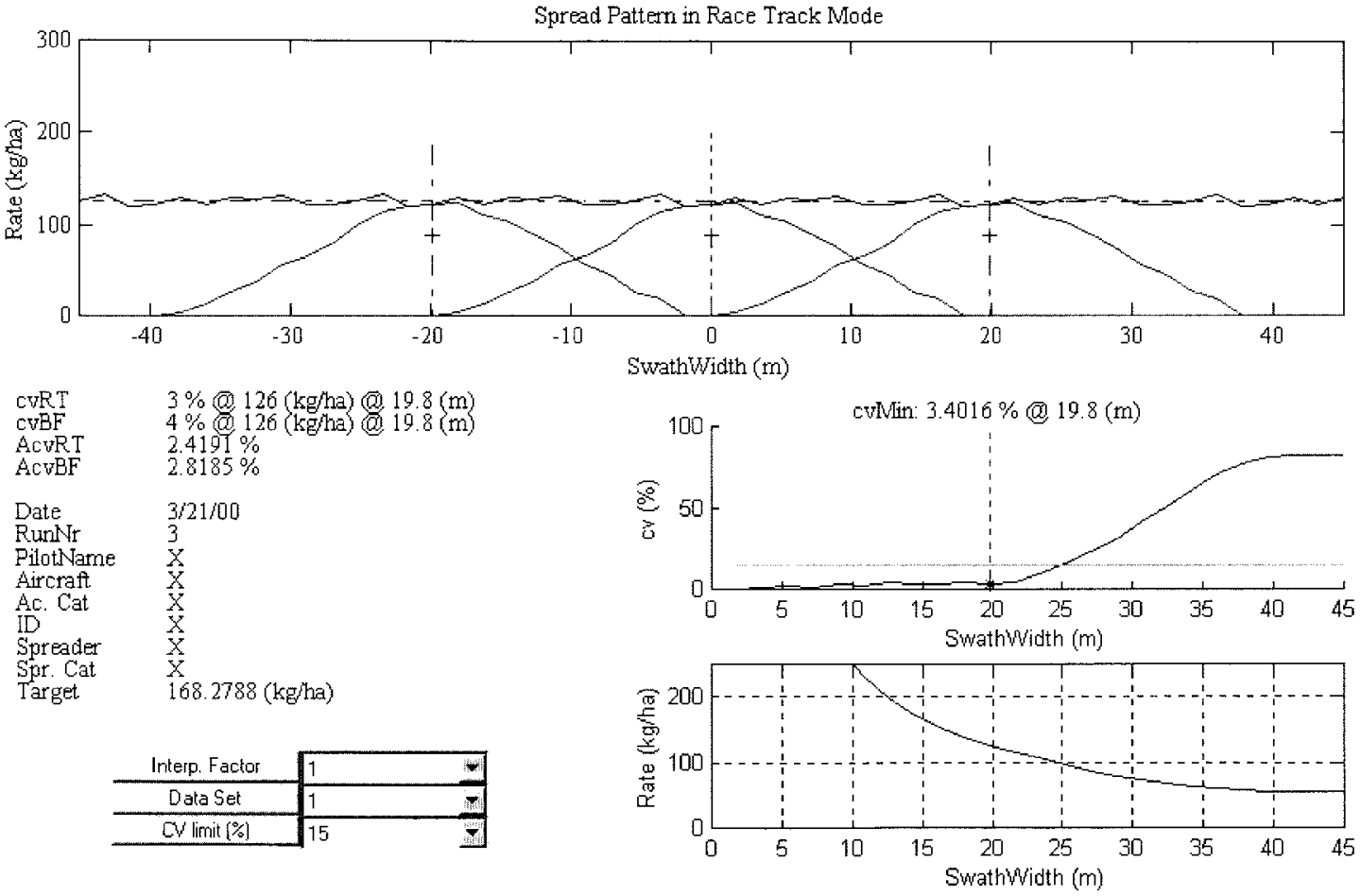

Figure 4(d)-Characteristics of a smooth triangular spread pattern (RaceTrack, optimal uniformity/swath width combination). 

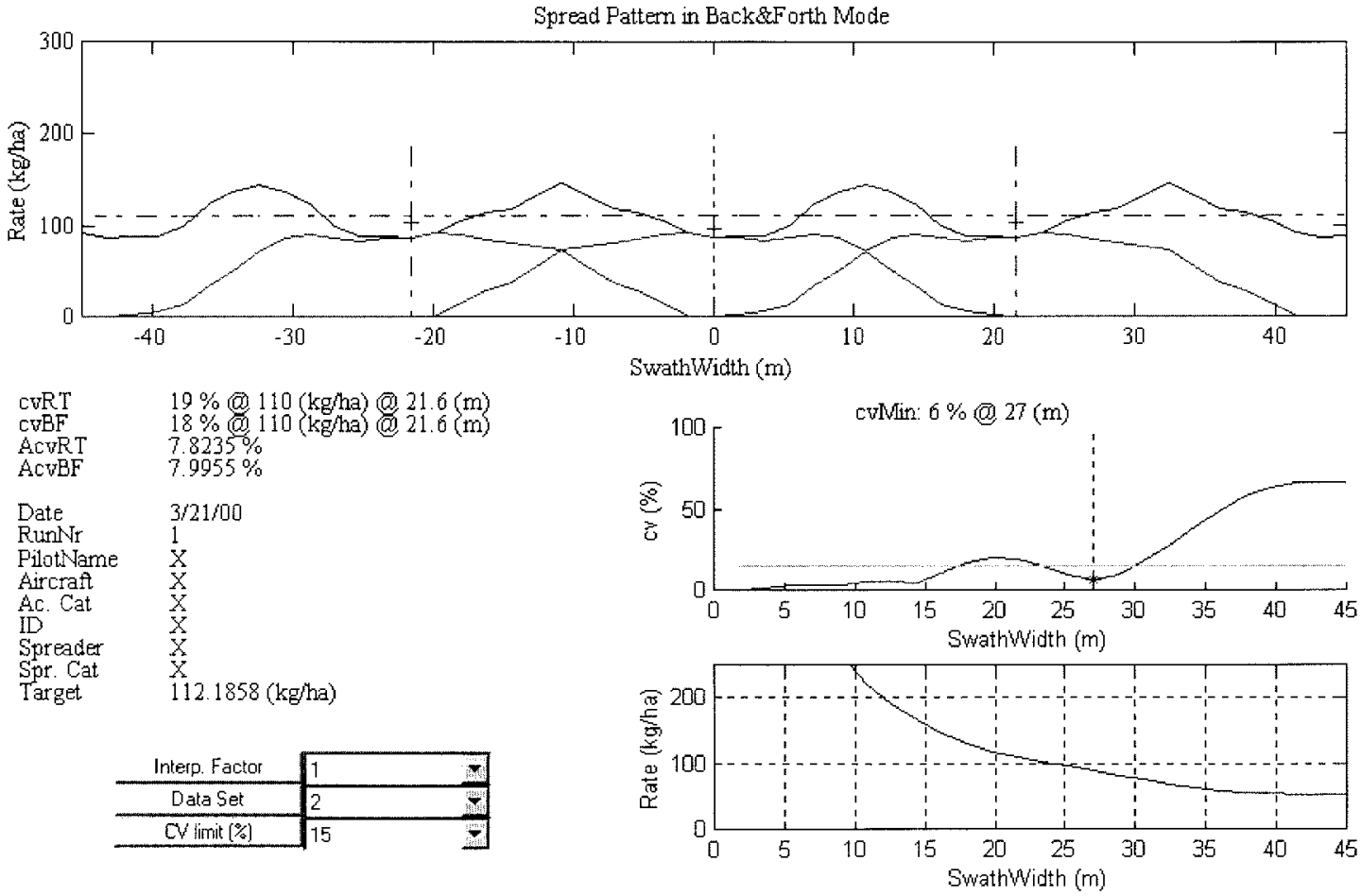

Figure 5(a)-Characteristics of a wide trapezoidal spread pattern (Back\&Forth, rate close to target rate).

Files Simulation Preferences Made Help
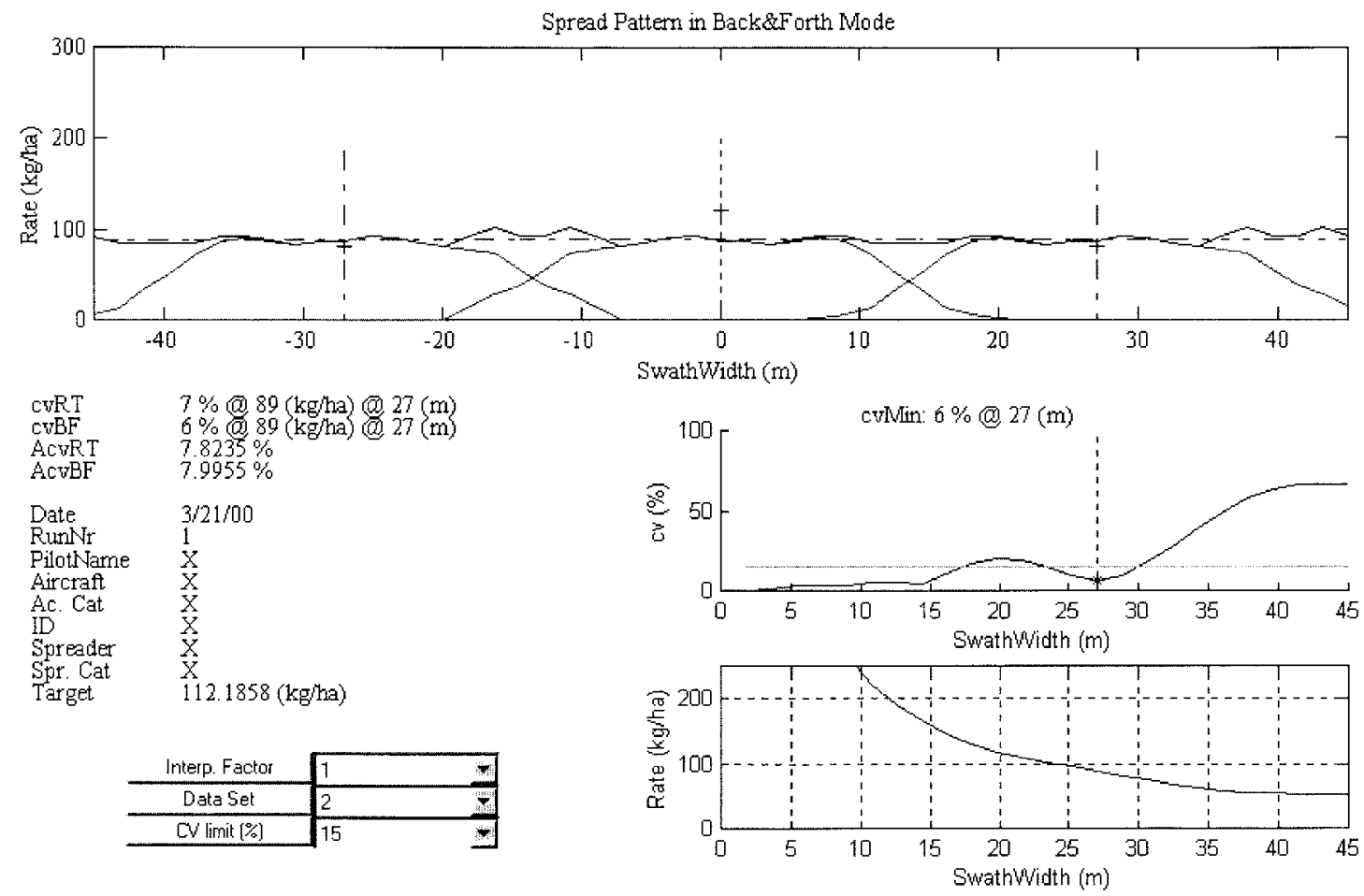

Figure 5(b)-Characteristics of a wide trapezoidal spread pattern (Back\&Forth, optimal uniformity/swath width combination). 
Eles Simulation Preferences Mode Help
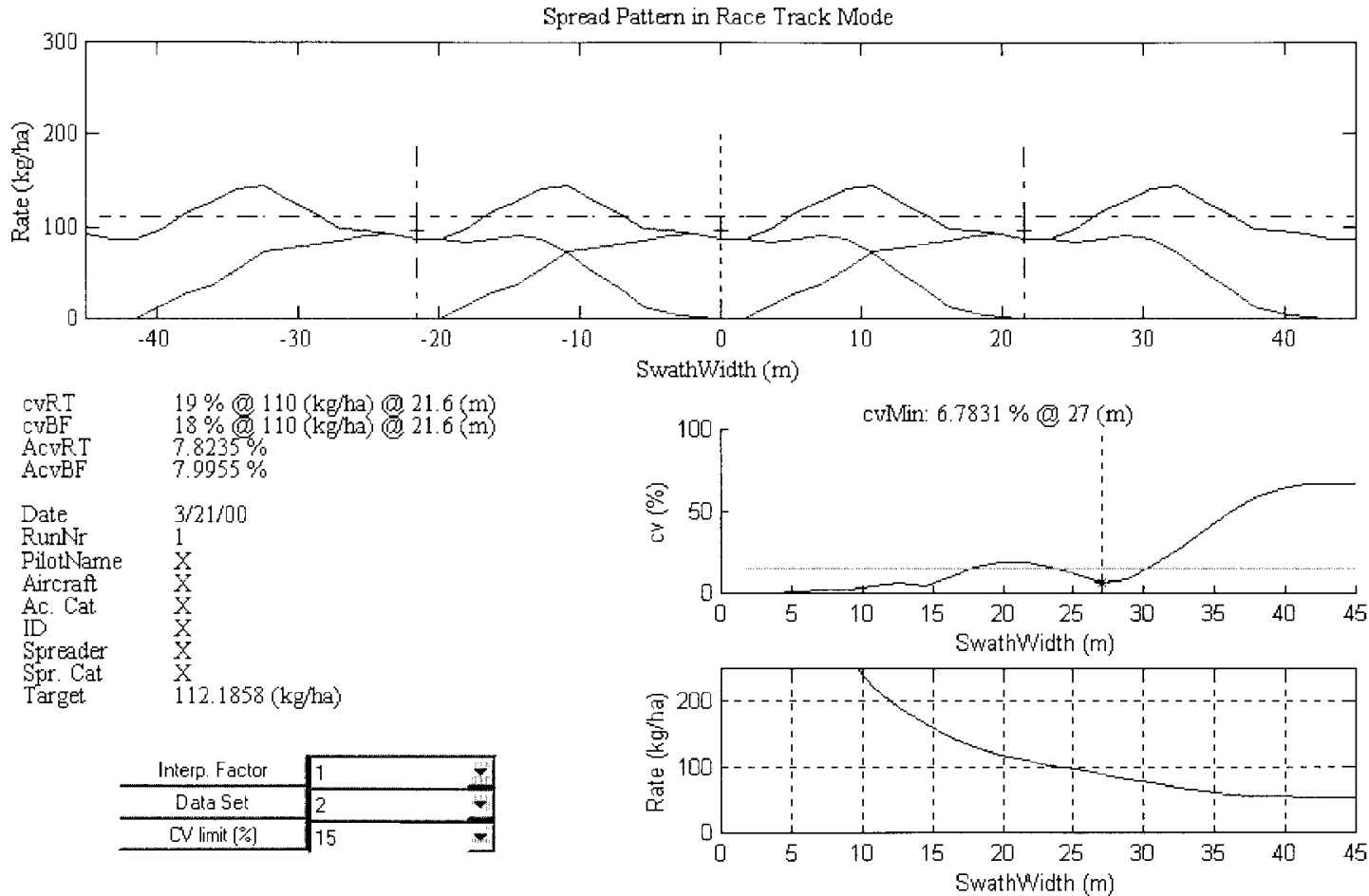

Figure 5(c)-Characteristics of a wide trapezoidal spread pattern (RaceTrack, rate close to target rate).

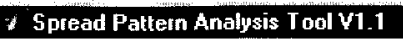

Files Simulation Ereferences Mode Help
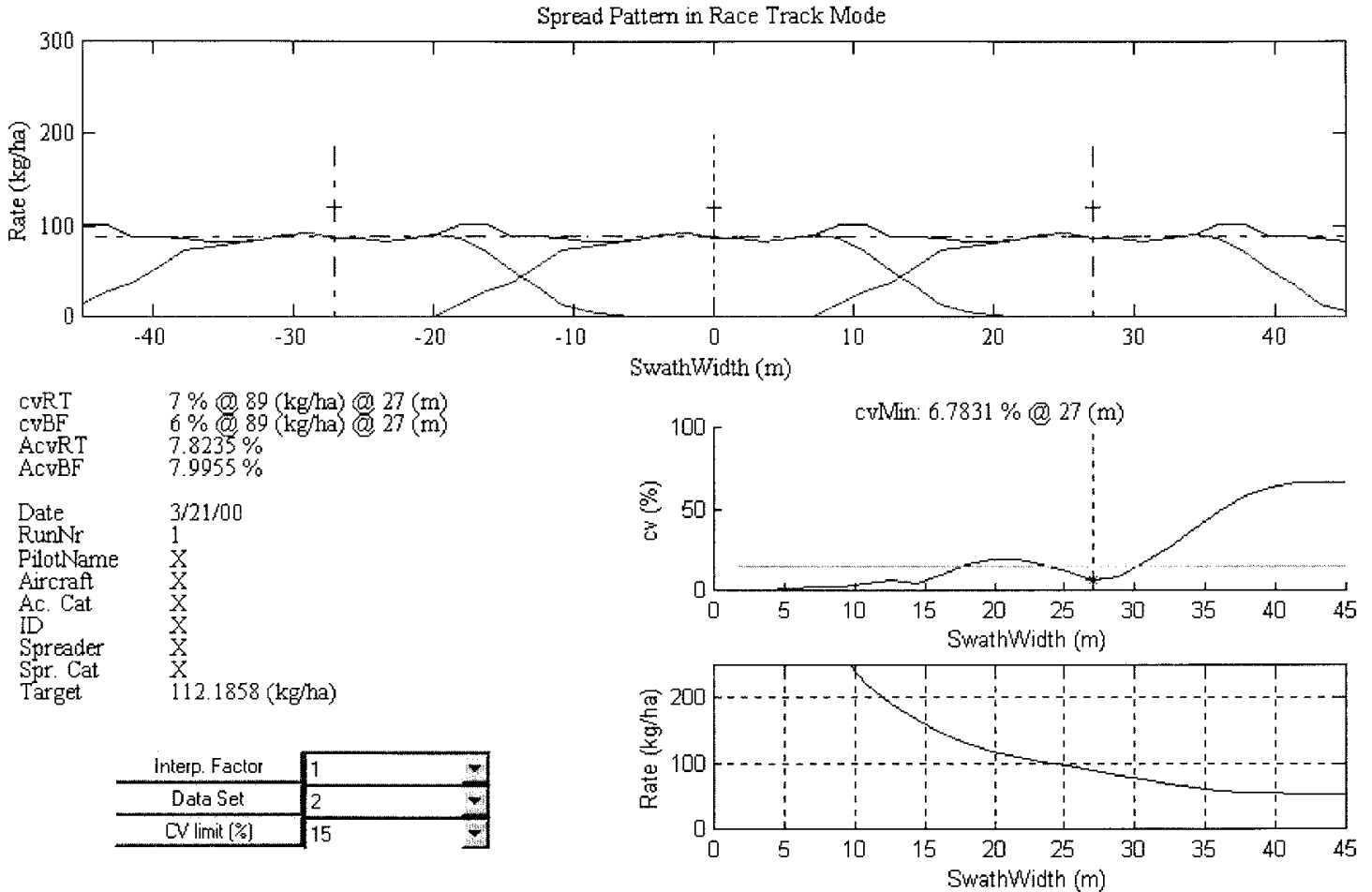

Figure 5(d)-Characteristics of a wide trapezoidal spread pattern (RaceTrack, optimal uniformity/swath width combination). 

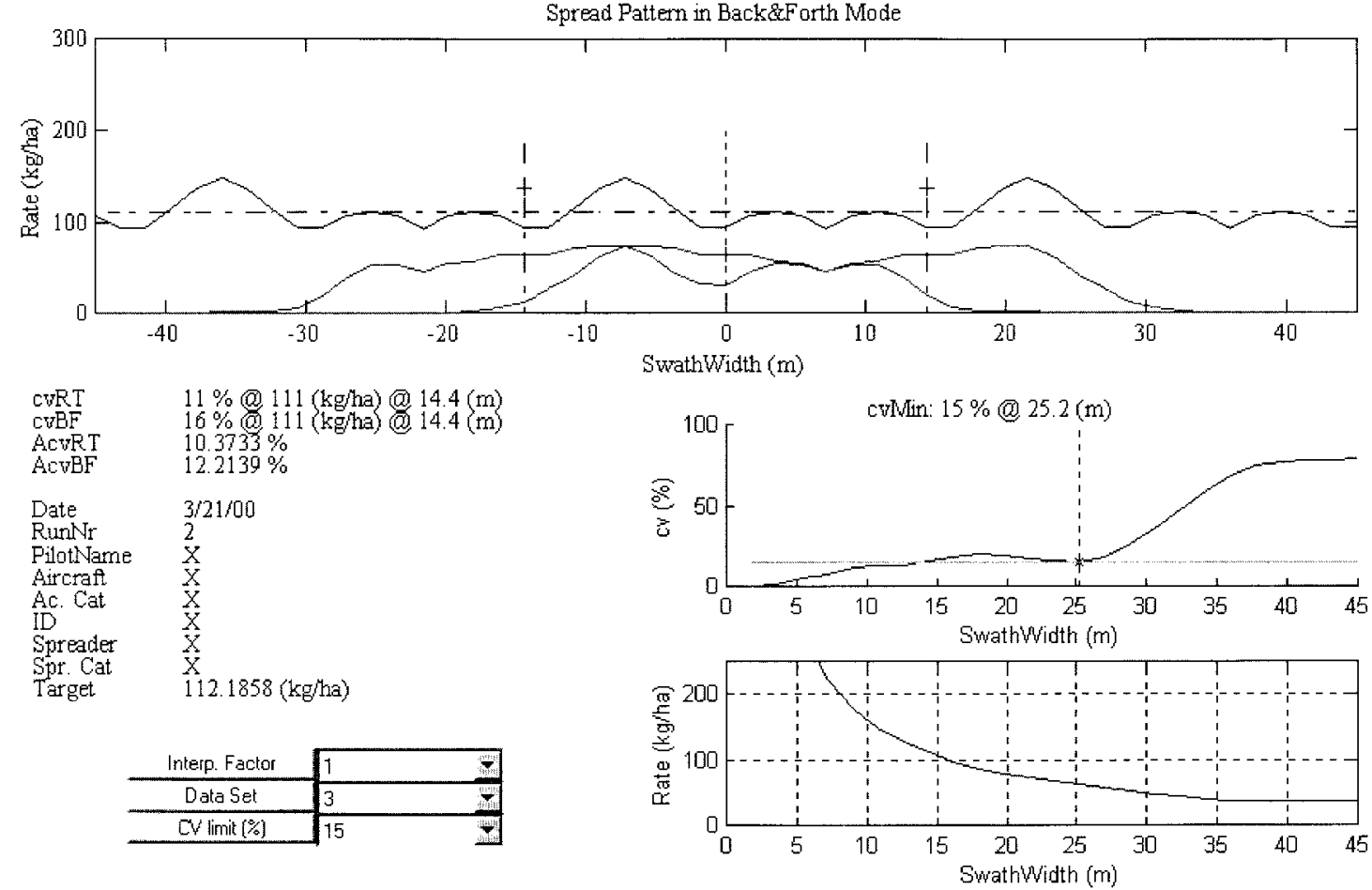

Figure 6(a)-Characteristics of a skewed trapezoidal spread pattern (Back\&Forth, rate close to target rate).

Files Sinulation Preferences Made Help
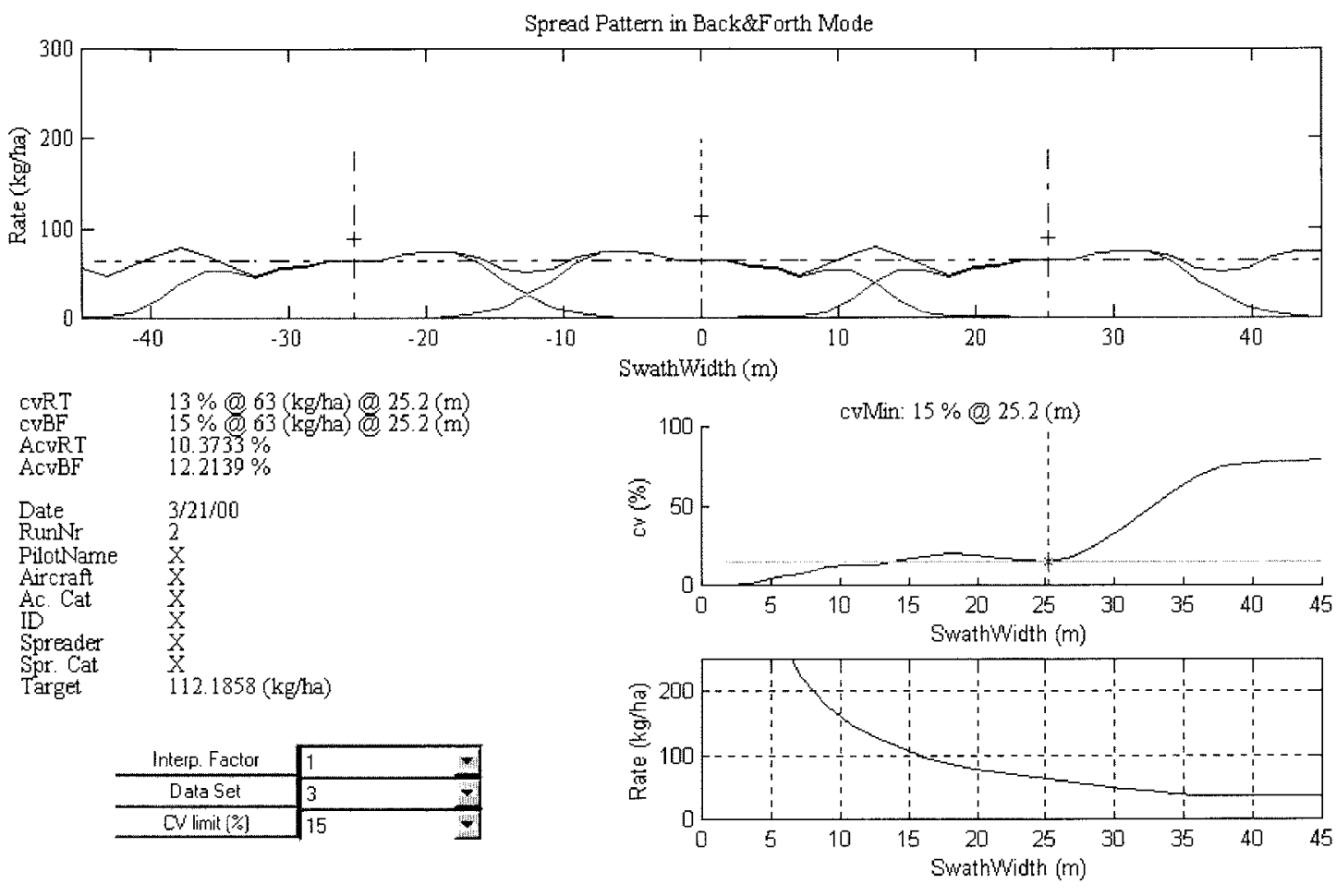

Figure 6(b)-Characteristics of a skewed trapezoidal spread pattern (Back\&Forth, optimal uniformity/swath width combination). 
Files Simulation Ereterences Mode Help
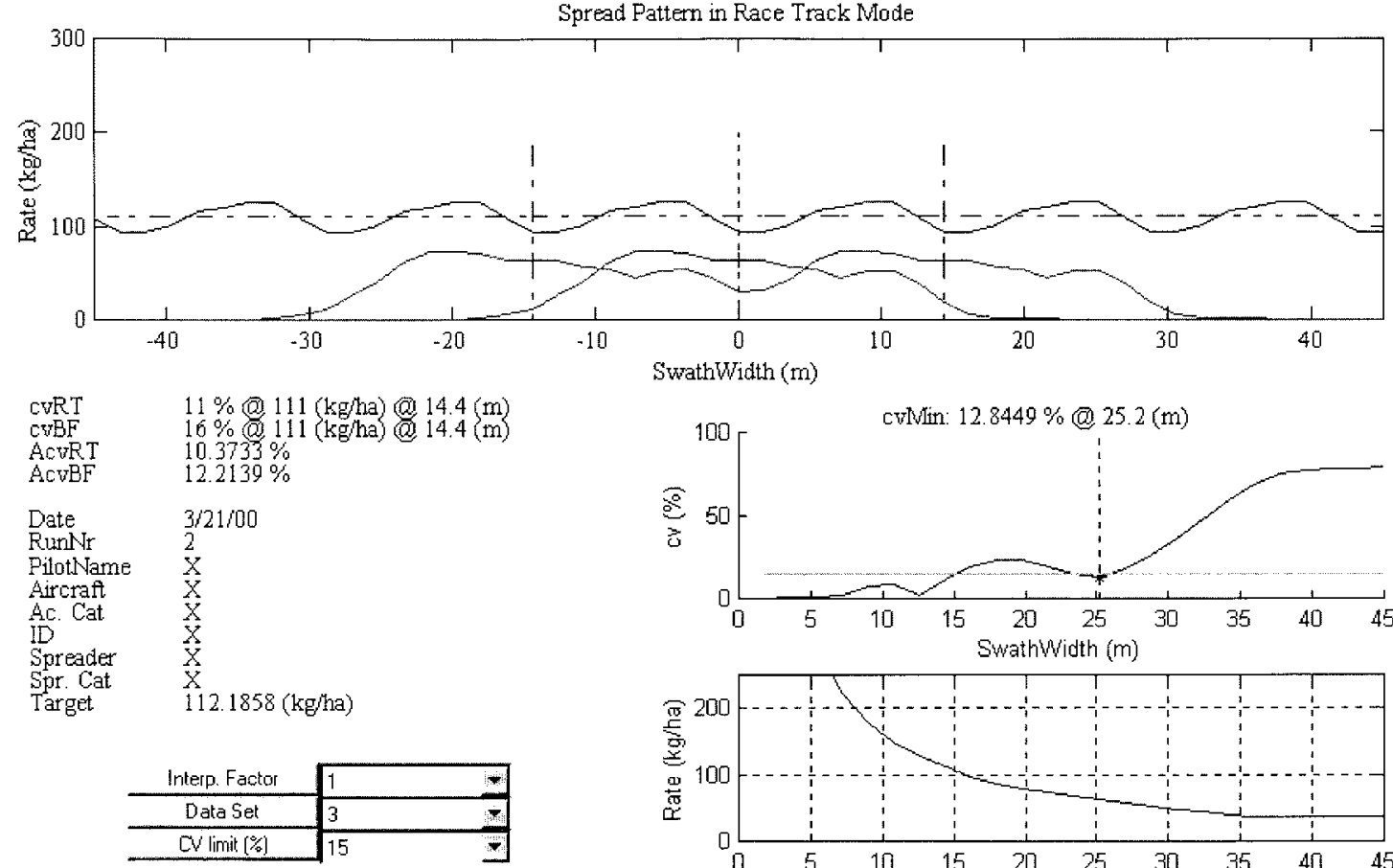

SwathWidth $(\mathrm{m})$
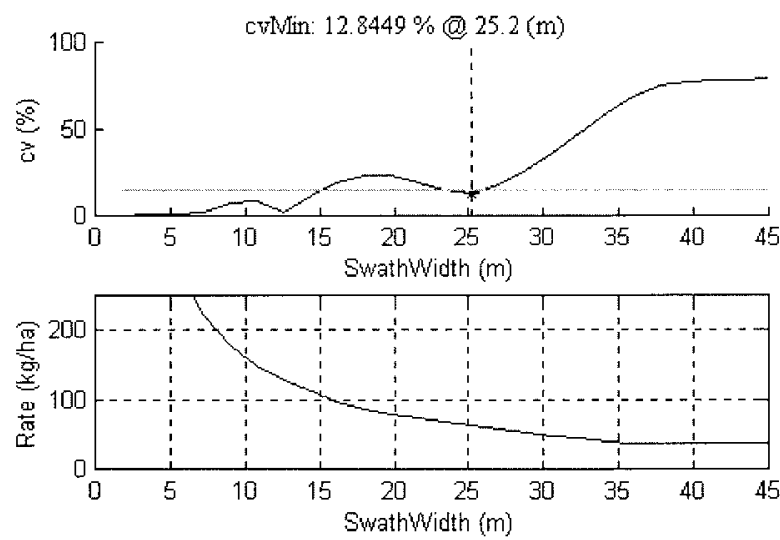

Figure 6(c)-Characteristics of a skewed trapezoidal spread pattern (RaceTrack, rate close to target rate).
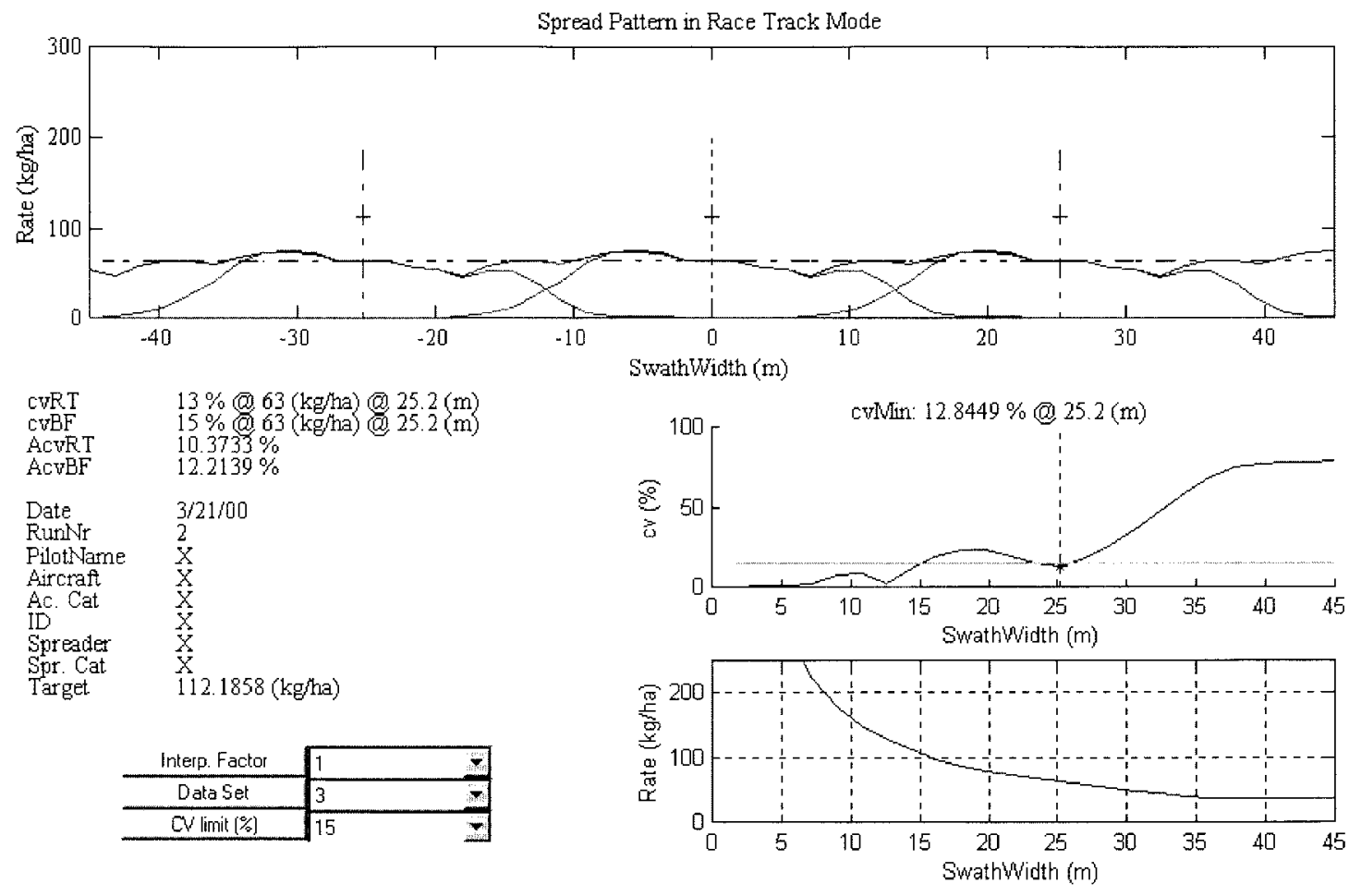

Figure 6(d)-Characteristics of a skewed trapezoidal spread pattern (RaceTrack, optimal uniformity/swath width combination). 

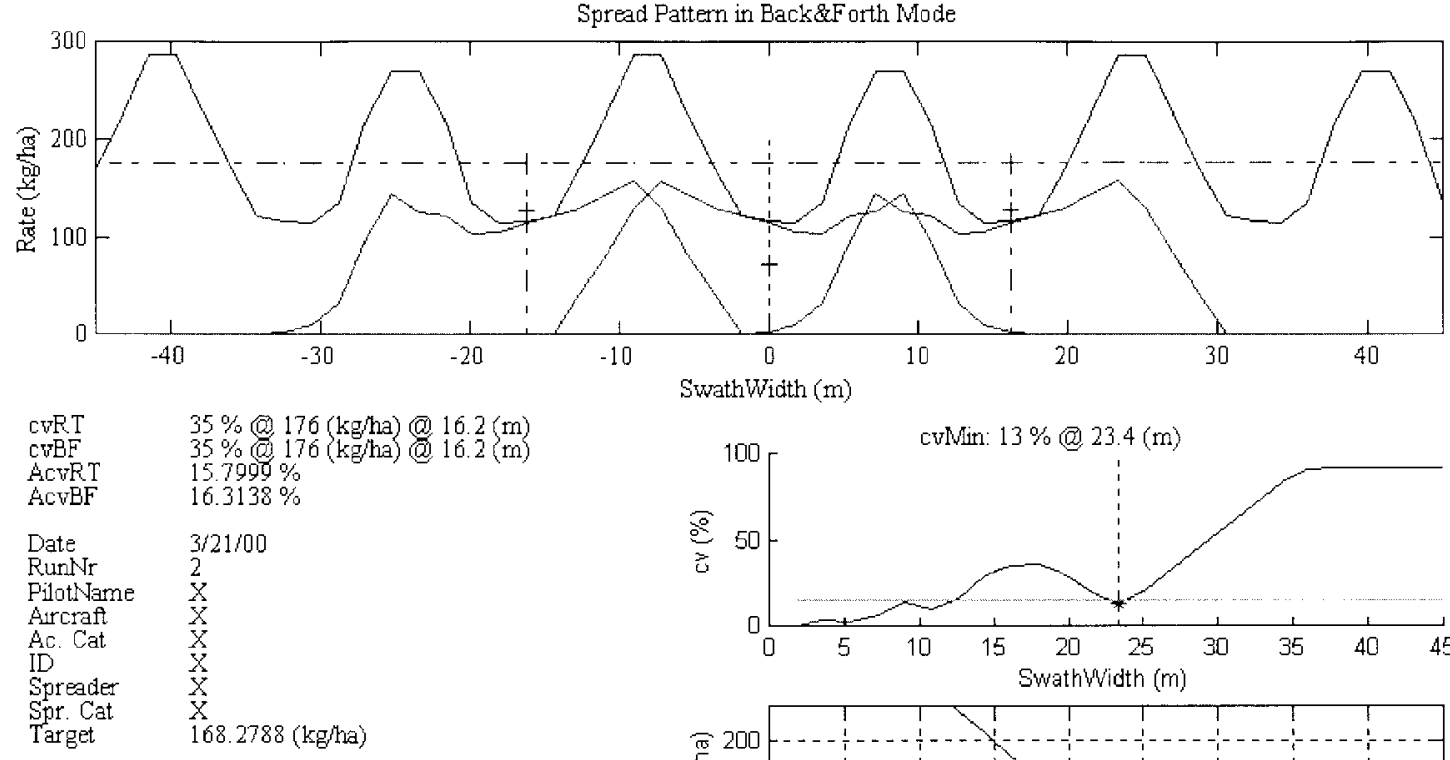

SwathWidth (m)

\begin{tabular}{c|ll} 
Interp. Factor & 1 \\
\hline Data Set & 4 & \\
\hline CV limit (\%) & 15 & \\
\hline
\end{tabular}
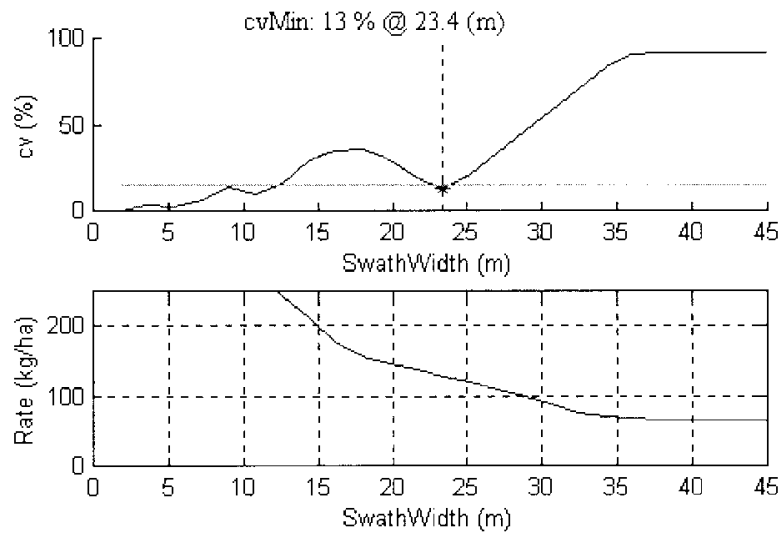

Figure 7(a)-Characteristics of an M-shape spread pattern (Back\&Forth, rate close to target rate).

\section{Spread Pattem Analysis Tool V1.1}

\section{Files Simulation Prelerences Mode Help}
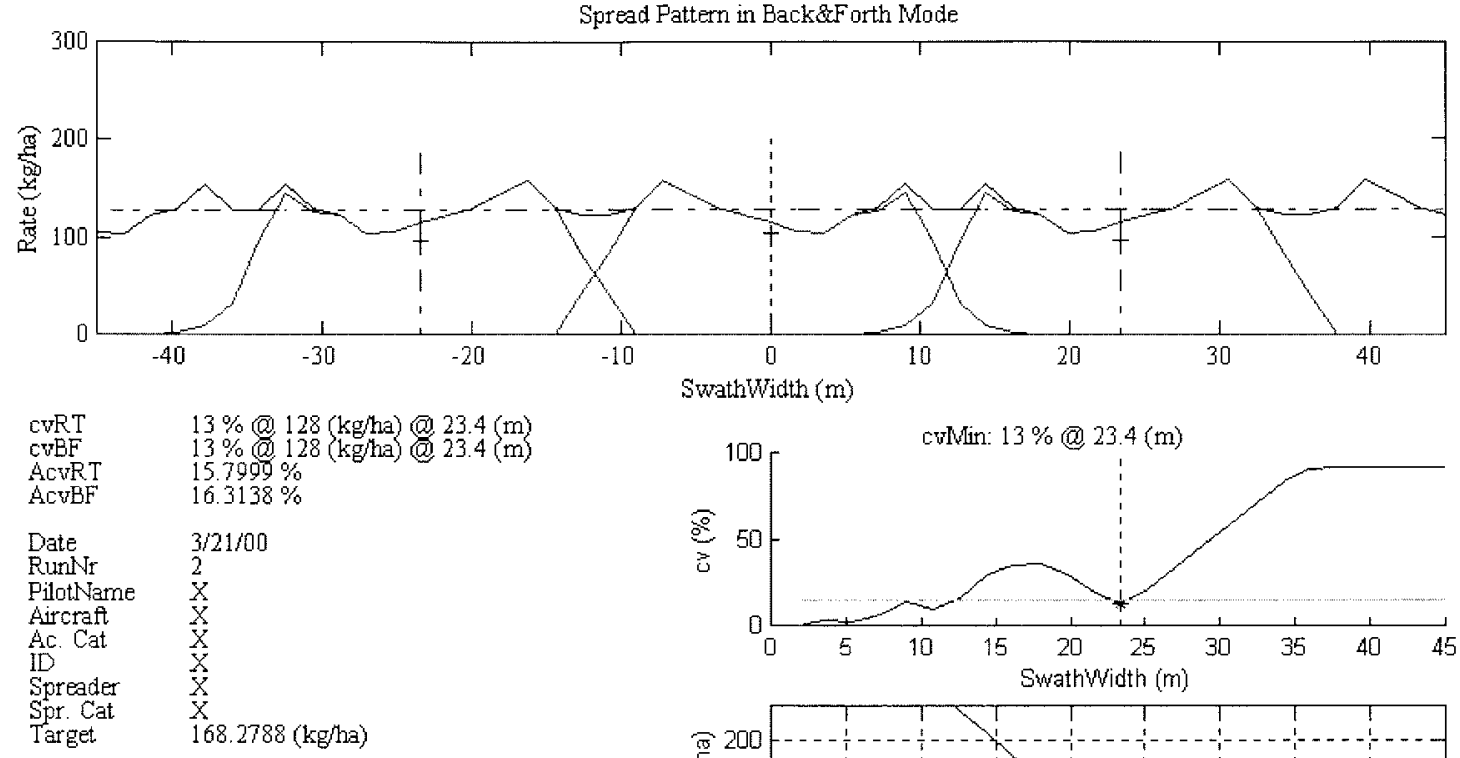

Target $168.2788(\mathrm{~kg} / \mathrm{ha})$

\begin{tabular}{c|ll} 
Interp. Factor & 1 \\
\hline Data Set & 4 & \\
\hline CV limit (\%) & 15 & \\
\hline
\end{tabular}

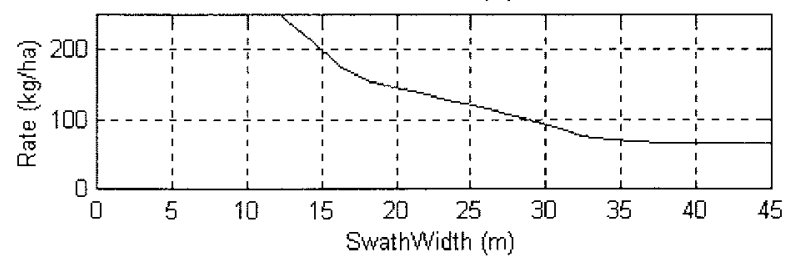

Figure 7(b)-Characteristics of an M-shape spread pattern (Back\&Forth, optimal uniformity/swath width combination). 
Spread Pattern Analysis Tool V1.1

Eiles Simulation Pieferences Mode Heip
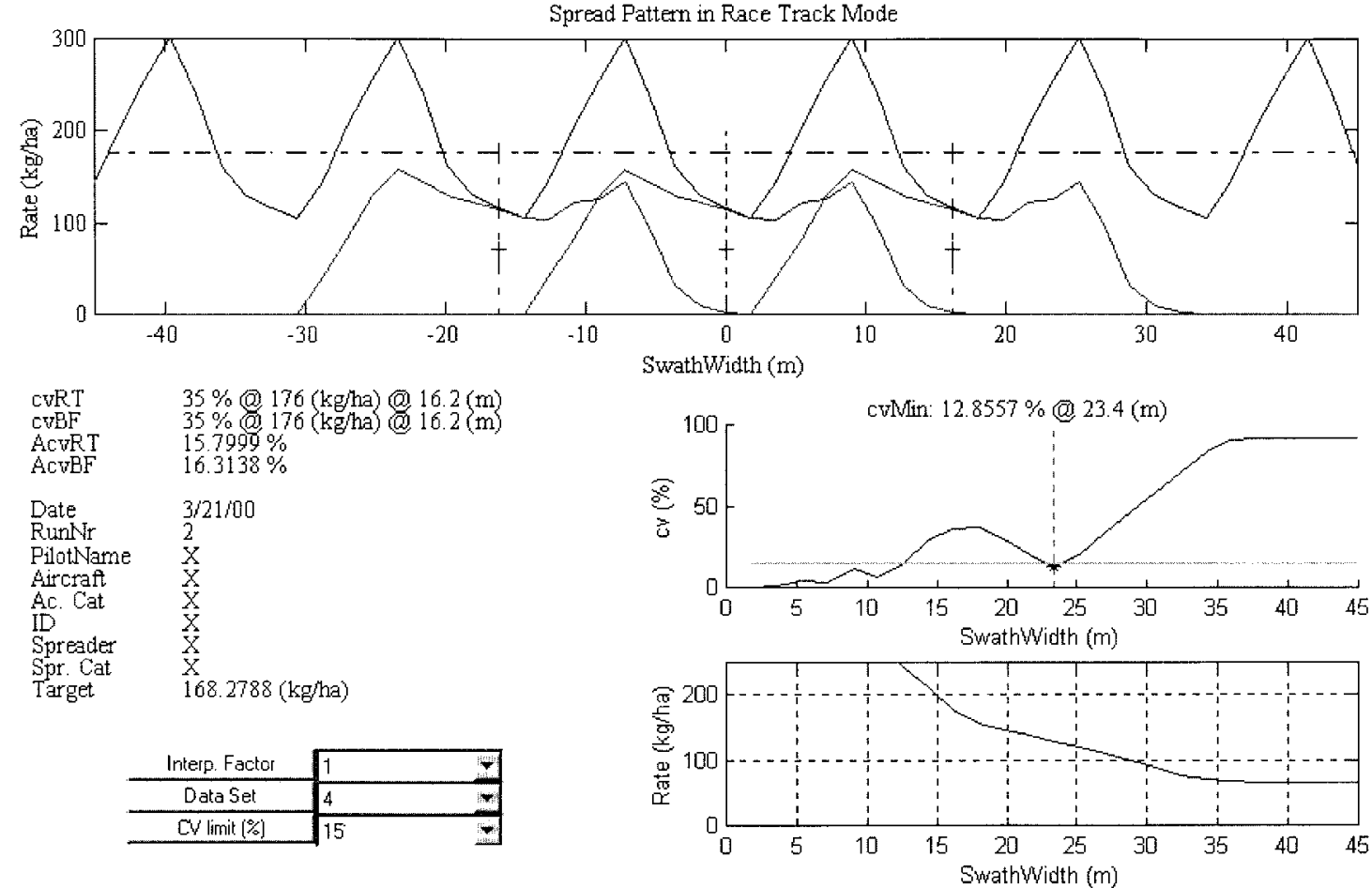

Figure 7(c)-Characteristics of an M-shape trapezoidal spread pattern (RaceTrack, rate close to target rate).

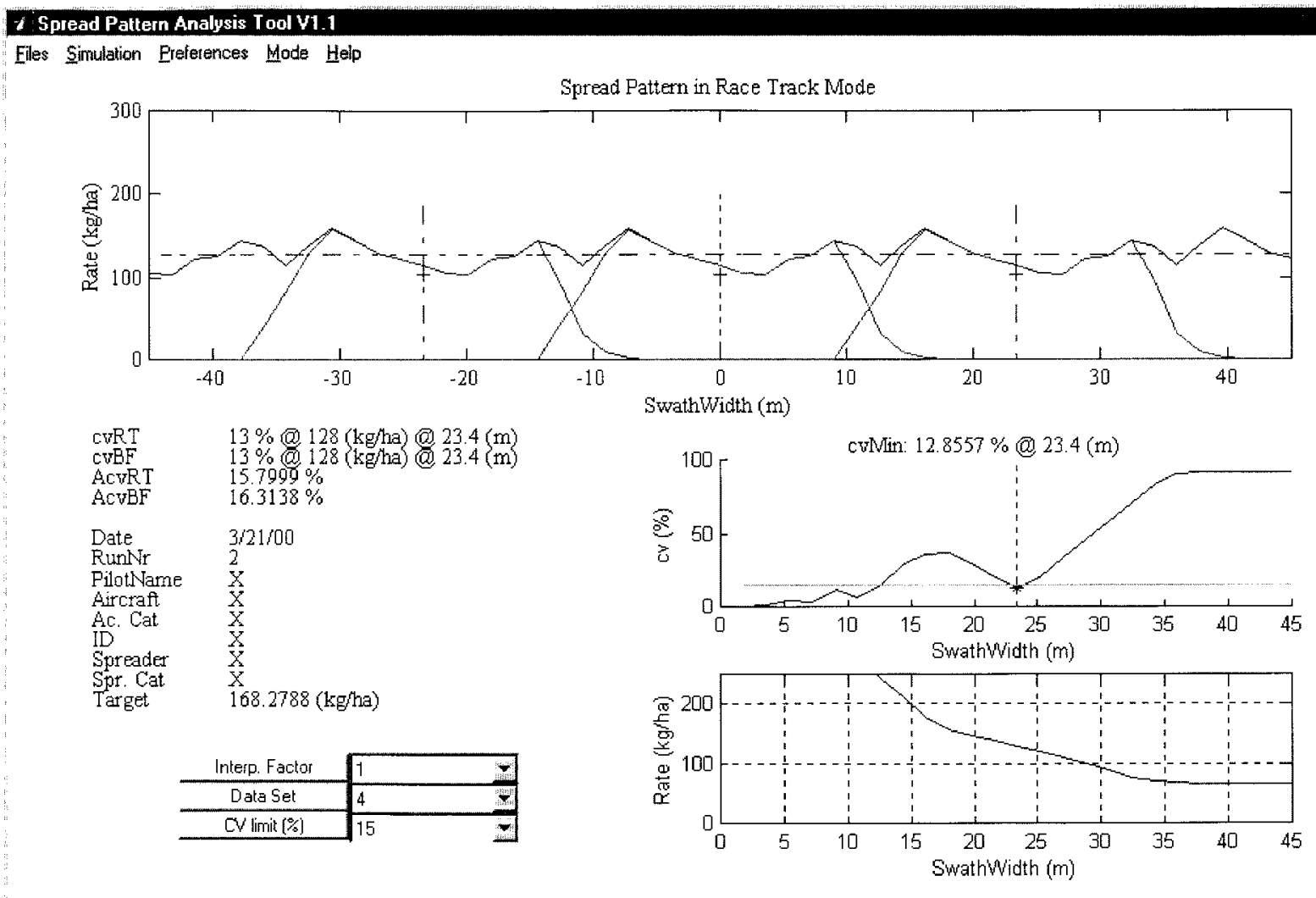

Figure 7(d)-Characteristics of an M-shape spread pattern (RaceTrack, optimal uniformity/swath width combination). 
Table 1. Relative distribution of pattern robustness for initial (Run 1) and adjusted (Run 2) test runs in Back\&Forth flight mode (470 test runs)

\begin{tabular}{ccc}
\hline & Run 1 $(\%)$ & Run 2 $(\%)$ \\
\hline AcvBF $<5$ & 3.45 & 2.75 \\
$5<$ AcvBF $<10$ & 32.57 & 34.86 \\
$10<$ AcvBF $<15$ & 39.85 & 39.91 \\
$15<$ AcvBF $<20$ & 15.71 & 16.51 \\
$20<$ AcvBF $<25$ & 6.51 & 5.05 \\
$25<$ AcvBF $<30$ & 1.53 & 0.92 \\
$30<$ AcvBF & 0.38 & 0.00 \\
\hline
\end{tabular}

(1997) used a computer program called AGDISP (Walker and Gardisser, 1989) and found that the deposition pattern of a typical 11-duct, wide tail spreader has three distinct high rate zones separated by two gaps of low rates, in effect producing an M-shaped pattern. The spreader was assumed to have nominal settings for inlet distances between the vanes (unadjusted), and equal material flows into the ducts (perfect gate behavior).

As in the previous case, there is a swath width at which the pattern can produce acceptable uniformity (at $23.4 \mathrm{~m}$ ), with cvs of 13 (Back\&Forth mode) and 12.8\% (RaceTrack mode). Apart from the fact that the rate is completely off here $\left[128\left(\mathrm{~kg} \mathrm{ha}^{-1}\right)\right.$ compared to the $168\left(\mathrm{~kg} \mathrm{ha}^{-1}\right)$ target rate], small deviations from the 23.4 swath width flight path will result in unacceptable uniformity. Producing a uniform spread pattern is simply impossible with an M-shaped pattern.

The robustness factors AcvBF and AcvRT are 16.31\% and $15.80 \%$, respectively, as in the previous case, both are higher than $5 \%$. This pattern is non-robust in both flying modes and should be avoided at all times.

\section{Robustness of Spread Patterns}

All spread runs performed at calibration workshops have a run number in the data set. The first run is done to see whether adjustments are necessary. The second run is usually after adjustments have been made to the spreaderaircraft combination. By comparing the robustness of first and second runs it was determined whether adjustment did have a positive effect on robustness. A total of 470 first and second runs were included in the assessment.

Robustness of Patterns in Back\&Forth Mode. The spread pattern robustness distribution of 470 test runs in Back\&Forth mode (AcvBF) is shown in table 1. It is clear that the percentage of robust spread patterns is very low. The majority of patterns (around 70\%) had an AcvBF between $5 \%$ and $15 \%$. When the first and second runs were compared for Back\&Forth mode, adjustments seemed to have a negative effect on some initially robust patterns. This suggests that good performing equipment was adjusted, resulting in worse patterns, although the difference in percentage was too small to conclude this with certainty. Overall, there does not seem to be any significant improvement in robustness between Run 1 and Run 2.

Robustness of Patterns in RaceTrack Mode. The distribution of the average cv for RaceTrack mode (AcvRT) is shown in table 2. Here, the patterns show that after adjustments (Run 2) a mere $10.28 \%$ is robust. As in the Back\&Forth mode, the majority (around 70\%) of patterns had an AcvRT between 5 and 15\%.
Table 2. Relative distribution of pattern robustness for initial (Run 1) and adjusted (Run 2) test runs in RaceTrack flight mode (470 test runs)

\begin{tabular}{ccc}
\hline & Run 1 $(\%)$ & Run 2 $(\%)$ \\
\hline AcvRT $<5$ & 8.59 & 10.28 \\
$5<$ AcvRT $<10$ & 30.86 & 34.11 \\
$10<$ AcvRT $<15$ & 38.67 & 35.05 \\
$15<$ AcvRT $<20$ & 14.45 & 18.69 \\
$20<$ AcvRT $<25$ & 5.08 & 1.87 \\
$25<$ AcvRT $<30$ & 2.34 & 0.00 \\
$30<$ AcvRT & 0.00 & 0.00 \\
\hline
\end{tabular}

As a comparison, high quality pattern shapes that have robustness factors in the $0 \%$ to $5 \%$ range are Gaussian shapes (Bell curves), triangles, and narrow trapezoids. Low quality shapes with robustness factors in the 5\% to $15 \%$ range are "shouldered" patterns (wide trapezoids), skewed patterns, and M-shapes (Grift, 2000).

\section{SUMMARY AND CONCLUSIONS}

A new definition called robustness was used to express the quality of a spread pattern. In contrast to traditional rate and cv measures, robustness takes the whole cv-swath width relationship into account. It was defined as the average $\mathrm{cv}$ of a pattern for swath widths in the range of zero to the minimum $\mathrm{cv}$ at the largest swath width. All analyses were performed using a Spread Pattern Analysis Tool (SPAT), a custom developed Windows-based program (Grift, 2000).

From studying real spread patterns, the following conclusions were drawn:

1. A good spread pattern is symmetric, has the target rate in the center and should monotonically decrease at both sides of the center (Gaussian, triangles).

2. A narrow trapezoidal shape is acceptable, as long as the horizontal leg of the trapezoid is smaller than one-third of the total spread width (critical trapezoid). Under certain conditions, the critical trapezoid can easily transform into an M-shape with potentially detrimental effects on the uniformity.

3. Too much emphasis on wide spread widths can result in low quality spread patterns. Patterns that are too shouldered (wide trapezoids) exhibit a swath width region where the uniformity is unacceptable which makes them non-robust.

4. M-shaped patterns, or, in general, any pattern shape that does not monotonically decreases at both sides of the center, make producing a robust overlapped pattern virtually impossible.

5. Skewness seems to increase the $\mathrm{cv}$ for all useful swath widths. It has a very negative effect, particularly in Back\&Forth flight mode. Sources of skewness are poorly adjusted spreader vanes and more importantly, gates that do not open evenly. Another source of skewness is side wind. Skewness should be avoided as much as possible by periodical calibration of the spreader/aircraft combination.

The overall robustness of the spread patterns analyzed, was found to be very low (approximately $10 \%$ of 470 patterns in RaceTrack and approximately $3.5 \%$ in Back\&Forth mode were robust). The majority of patterns had a robustness factor in the range of $5 \%$ to $15 \%$, which 
places them in the categories of shouldered patterns (wide trapezoids), skewed patterns, and M-shapes. The magnitude of the problem is such that periodic calibration alone will not significantly improve the robustness of spread patterns. New and redesigned equipment and spreading procedures may be necessary to accomplish this.

ACKNOWLEDGMENTS. The authors would like to thank the ground crews, extension personnel and all pilots that contributed to the collection of the data that made this exercise a valuable one.

\section{REFERENCES}

ASAE Standards, 46th Ed. 1999. S386.2. Calibration and distribution pattern testing of agricultural aerial application equipment. St. Joseph, Mich.: ASAE.

Bansal. R. K. 1997. Computer simulation of granular material flow in aerial spreaders. Ph.D. diss. Fayetteville, Ark.: University of Arkansas.

Gardisser, D. R. 1993. Agricultural aircraft spreader setup. Pub. MP 351. Little Rock, Ark.: Cooperative Extension Service, University of Arkansas.
Grift, T. E. 2000. Spread Pattern Analysis Tool (SPAT): 1. Development and theoretical examples. Transactions of the ASAE 43(6): 1341-1350.

Helms, R. S., T. J. Siebenmorgen, and R. J. Norman. 1987. The influence of uneven preflood nitrogen distribution on grain yields of rice. Arkansas Farm Research, University of Arkansas, March-April.

MatLab, Ver 5.3. 1999. Natick, Mass.: The Math Works Inc.

Parish, R. L. 1987. A computer program for spread pattern analysis. Applied Engineering in Agriculture 3(1): 14-16.

. 1999. The effect of spreader fill level on delivery rate. Applied Engineering in Agriculture 15(6): 647-648.

Walker, J. T., and D. R. Gardisser. 1989. Using AGDISP for dry material deposition analysis. ASAE Paper No. AA89-006. St. Joseph, Mich.: ASAE. 
